\title{
Study on the Database Security Technology in E-commerce Environment
}

\author{
Li Yang \\ School of business, Northwest Normal University, Lanzhou Gansu, 730070, China
}

Keywords: E-commerce, Database, Security technology, Solution.

\begin{abstract}
Today, e-commerce gain the people's attention, become an indispensable part of life, but also made a contribution for the development of world's economic the security of e-commerce website is the foundation to ensure the smooth running of the website, the guarantee on the security of the database is a systematic problem. This paper analyzes the issues and causes of the database security technology in the e-commerce environment, and puts forward the solutions to solve this problem.
\end{abstract}

\section{Introduction}

In E-commerce network is between both side of the website instead of the traditional face to face communication, connection and transactions, many uncertainty factors rising up, coupled with the threatened network database security, environment for e-commerce will be more severe, it will hinder the development of economic and social progress. Thus, security of database issues in e-commerce environment cannot be ignored, more and more importance and research conducted on this topic. This paper discusses and analyses the causes of the issues and the solution for them

\section{The security issues for database under the e-commerce environment}

In the rapid development of economic in the 21st century, online shopping has become a part of people life indispensable, e-commerce activities won the common recognition of businesses and consumers, has brought great convenience to people's lives, e-commerce has authentication, confidentiality, integrity, availability, non repudiation ${ }^{[1]}$ However, the more rapid development of new things, the more complex for the legacy or others related problems will be, the more intense demand will needed for correct understanding and solutions to provident it from happening again。 Referring to many domestic and aboard literatures, the definition of e-commerce is to trading activities combined the producers, sellers and consumers together in the network by electronic technology. After discussion and analysis the author summed up that the main security problems for database under the e-commerce environment are faultiness database system, simplistic running system for the website and backward hardware facilities, the reason for these three issues are as flowing

\section{Faultiness database systems}

The perfection degree of database is the determine factor for the security of the e-commerce website. Database is the strong guarantee for the smooth running of the system. Most e-commerce website adopted DBMS system, which including subsystem like DBF、Ex-cel、MS SQL Server、 MYSQL 、ACCESS, etc. most of them can be decoded easily, several is easy to be copied, ${ }^{[2]}$ MS SQL server is the most common used one, that belongs to a large relational DBMS, compare to others it do have certain advantages, while because of the large number of user made some loopholes to the system Due to large number of user, its vulnerabilities will be more easily under attackers and take improper use of it, once the attacker made SA permissions, they will build their own database, and gain the authority access to the database; The attackers can perform storage process to run the program under Windows environment, as in the use of their own accounts, random changes to the data, delete information, start or close an activity. In those conditions there is no security to the database in the e-comer environment, so a improvement is urgently needed. 


\section{Simplistic running systems}

UNIX 和 LINUX 。Web operating system is usually called as running system, the most well-known one is the series of windows system, in addition to the huge user system, and other two systems that is rarely used, respectively is: Unix and Linux. Again the number of users is imbalanced divided between three of them, too much in the first one and too little for last two, the system vulnerability is more likely to be found by hackers, followed by the constant attack, or implanted Trojan virus. This will make the system paralysis, information leakage, the entire e-commerce environment will be even worse, more not everyone was convinced, and then to the decadence. If there is only small differences between the number of users for the three operation system, then it may not that easy to find the vulnerability of the system itself, we have These three major operating systems in the majority of users in the proportion of the use of a small difference, then it is not so easy to find the vulnerability of the system itself, the guaranteed. Security of the database can go further

\section{Backward hardware facilities}

With the rapid development of e-commerce, the backward of hardware facilities will become a stumbling block for it. server for the E-commerce website is what the combination workgroup of PC $\mathrm{s}$, which is made by the workgroup and at departmental level, some of them combined database and web server together to provide space for the network service providers, this combination is dangerous, because if the database or server receives is damaged, the attacker is likely to get administrative right, then the database will totally lost the security, so poor hardware like human without skeleton, hard to molding, security of the database of the network in e-commerce environment is the basic problem of protection.

\section{Reasons for the safety issues of database in the e-commerce environment}

The security problems for the database is very diverse, the reason is indescribable, since this is research article, author but between this is a research article, the following from the author take two aspects that personal considered it the most important to carry on the elaboration.

\section{The loopholes in the database system itself}

If the problems are basic operating system of the database, or in the database of web server vulnerabilities will be not allowed to produce illegal access, resulting in the destruction of the original data, the page can not normally access, the safe and orderly e-commerce activities will be upset ${ }^{[3]}$ The main reasons for that are: (1) each database has its own safety inspection system, and when the examination system is not timely upgrade or update, below the level of operating system or network system, database system cannot be accurately the of protection and update configuration; (2) The loopholes in the database system itself, is to open up a shortcut to the invaders for sure, equivalent to open the door and greet the guests. Attackers will gain highest authority of the system, change information, delete the content, Trojan is a cinch, direct threat to the security of operating system, resulting in paralysis of the system; (3) 。 Risk of software is another manifestations and reasons for the existence of loopholes in database. It is because the software did not install patches of the operating system; lock of full ranged security measures, so that it cannot more quickly to a dangerous situation to make analysis and measures.

\section{Trojan virus attacks on database}

Trojan virus attacking to database is a one of the large number of attackers in a way, but it can cause serious consequences. Trojan, also known as Trojan virus, is the virus that can conduct controlling from one computer another computer, usually bounded in the mail or information, once the recipient opened the website or the information, the Trojan will enter the computer and realize controlling, for example, the previous comparison of the classic panda burning incense. Because the network is running in an open environment, some of the agreement is no longer a secret, some attackers can intruder to the database through the network ${ }^{[4]}$, which also made a security flaws to the ecommerce or database. 


\section{Solutions for data security issues in the e-commerce environment}

Above analyzed and explored in detail about the database security problems and causes in the e-commerce environment, the following will focus on the up forwards solutions for the above mentioned problems, strive to make the network and database more secure and lay a good foundation for better public services to

\section{Establish and improve the database system, conduct full backup}

Establish a sound data backup and management regulations of the database are the necessary premise of ensuring the safety of the database. [5] But a detailed data files has to be established to ensure the performance system of recovery timely and effective, correct backup, it is the only way to prevent from adverse effects, moreover the correct way and the correct date for recovery and system must be used. Access to the relevant literature, for different database, there are 3 backup methods: only backup database, backup database and transaction log, incremental backup. Combined with the actual situation, the majority of enterprises will adopt is backup the database for comprehensive use, the actual operation condition and network security problems, the regular conversion of the three strategies have to be comprehensive considerate to conduct better contribute to the security of network database.

\section{Improve and updating the software}

Support network system is the two important components software and hardware, continuous improvement and upgrading of the hardware can effectively increase protection to the database security. This paper conducted the researches mainly from two aspects, the strengthening security level among the operating system and server and enhancing the security of database system of research, specific content see below.

Strengthen security level between the operating system and the server and upgrading security level of database itself, can effectively escort the security of database. when using the database, the need to strengthen the security level of the database itself, avoid the usage of default permissions. Set the account and password to a higher degree of security, password can be a combination of letters and numbers. this is the only to intensify protection of the account, the attacker will have difficulty to decipher the password; in the face of strong network hacker, this is completely inadequate, so time to time upgrading patches database will be needed to fix vulnerabilities, such layers of protection will minimize dangerous degree. If the operation of the database is relied on the operation of the computer network, the nature of the threat is from the virus invasion, so we should avoid viruses diffusion, destruction, hidden of the viruses through the network platform, it should be the effective prevention like, virus killing, supervision and management of governance methods to achieve upgraded database security. Numbers of data studies show that database software should use the SQL Server MS system, this system is the most suitable to meet the needs of users. in the use of the Database can be achieved by minimal the server window, it can turn off the unused ones in a timely manner, and increase the difficulty of the server port scan, by modifying the database server port. a series of standardized operations will be operated for the installation of the system, carrying out the security of database system from the source.

\section{Replace into some more reliable hardware}

Hardware, in fact, is relative to the software, is to ensure a level of physical security. There is also a fault-tolerant technique to ensure the security of the physical layer, such as the multi cluster system. Center server security is to focus on the protection of the firewall between the various systems, and locate sever inside of the firewall. Only authorized users can enter the firewall and network and access to information, otherwise have to be blocked outside the firewall, which is the best advantage for switching into more reliable hardware. It may be costly, but it is a good and effective way to assure the safety of the database in the e-commerce environment.

\section{Strengthen the website backstage management}

Strengthen the background management of the site, in fact, can improve work efficiency, and reduce the risk of network security: First, do not use simple user name and password with general rules in the backstage management, the fixed format in the system to is the administrator + password, if name and password is too simple or frequent used, it will lower the security of the system and 
increase the database information exposure risk; Second, of the home page cannot use the conventional naming method in the background of the management, it is not connected to the web page that other people can browse at any time. Third, set additional access to the web, to protect the information from leakage. When logging in, the authentication should not be ignored if there is any, because it is the only process to the verification can be done on the confirmation of a file and the next file browsing ${ }^{[6]}$ And a final possibility is the administrator did not withdraw from the system on the left, then it is inevitable to cause information leakage, the solution for this is to set a time period, like our screensaver, if there is no operation was done in a fixed period of time, the screen will automatically locked, and the permissions will be lapsed automatically, and need to log on again. This is a more intelligent way to prevent the leakage of our information, and protect our network database security.

\section{Conclusion}

Database security is the legacy of the rapid development of the modern society in the e-commerce environment of network. The main problems are faultiness database systems simplistic running systems and backward hardware facilities, simplistic running systems and backward hardware facilities, the reason for these kind $\mathrm{f}$ issues are The loopholes in the database system itself and Trojan virus attacks on database based on above mentioned problems and the causes, the corresponding solution are establish and improve the database system, conduct full backup, improve and updating the software, replace into some more reliable hardware and strengthen the website backstage management. The implementation of the four introduced solutions, it can future ensure the security of network database and preventing form series of acts of sabotage such as steal information by the attacker easily, delete the contents, Trojan horse virus and so on, and security escort for the database in the e-commerce environment. This initiative so that the public can be more relaxed in the face of e-commerce trading, communication, no longer nervous, fear of bad things happen all the time, for economic construction contributed to also improve the social efficiency, increase the trust between people. In this paper, the author conduct a comprehensive analysis and research on the database security technology problem system under the environment of e-commerce network, the author hope that through elaboration in this article can offer certain enlightenment function to solve the security problems of the existing network database.

\section{References}

[1] Xue Rui. Research on e-commerce security based on SET protocol, Zhengzhou University, 2013

[2] Zhou Ming. Analysis on Database security technology of E-commerce site, Software guide, 2010, 9(9509):52-53.

[3] Zhao Yuping, Wang Shuang. Research on Web database technology based on Technology in e-commerce environment, Information security and technology, 2013, 4(3703):54-56+60.

[4] Wu Yunying. Design and implementation of e-commerce based Delphi+PHP website security system, Hunan University, 2013.

[5] Zhang Hao. Analysis on technology databasesecurity of e-commerce website, Journal of Jilin Institute of economic management cadre institute, 2012, 26(13701):68-70.

[6] Li Guangjie. Research on Technology WEB databasesecurity in E-commerce environment, Computer CD software and application, 2014, 17(25423):29-30+33. 